\title{
Transplantation of human umbilical cord blood- derived mononuclear cells induces recovery of motor dysfunction in a rat model of Parkinson's disease
}

\author{
This article was published in the following Dove Press journal: \\ Journal of Neurorestoratology \\ 4 April 2016 \\ Number of times this article has been viewed
}

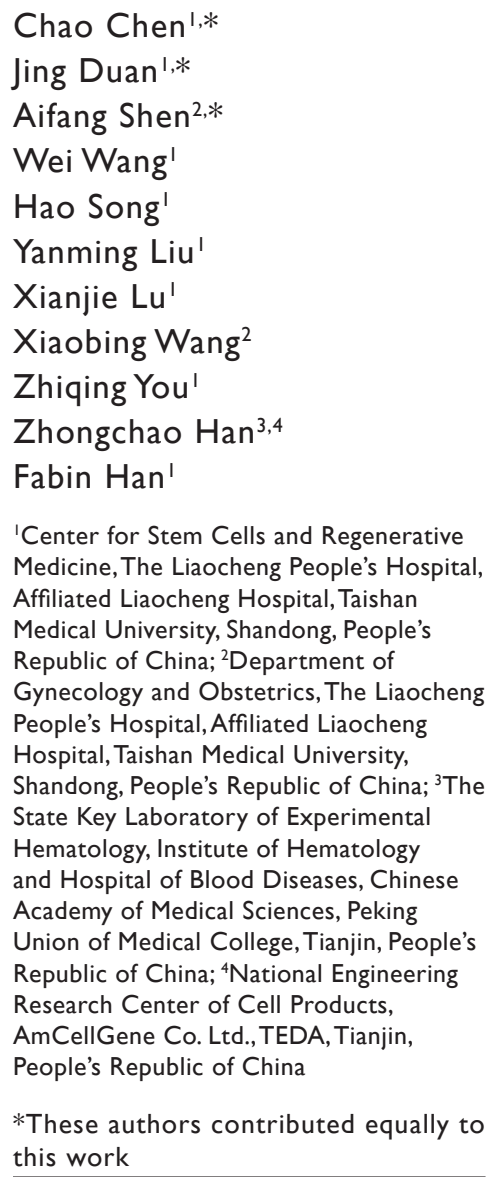

Correspondence: Fabin Han

Center for Stem Cells and Regenerative

Medicine, The Liaocheng People's Hospital,

Affiliated Liaocheng Hospital, Taishan

Medical University, 67 Dongchang West

Road, Liaocheng, Shandong 252000,

People's Republic of China

Tel +86 6358278427

Fax +86 6358272732

Email fhan2013@I26.com

\begin{abstract}
Human umbilical cord blood-derived mononuclear cells (hUCB-MNCs) were reported to have neurorestorative capacity for neurological disorders such as stroke and traumatic brain injury. This study was performed to explore if hUCB-MNC transplantation plays any therapeutic effects for Parkinson's disease (PD) in a 6-OHDA-lesioned rat model of PD. hUCB-MNCs were isolated from umbilical cord blood and administered to the striatum of the 6-OHDA-lesioned rats. The apomorphine-induced locomotive turning-overs were measured to evaluate the improvement of motor dysfunctions of the rats after administration of hUCB-MNCs. We observed that transplanted hUCB-MNCs significantly improve the motor deficits of the PD rats and that grafted hUCB-MNCs integrated to the host brains and differentiated to neurons and dopamine neurons in vivo after 16 weeks of transplantation. Our study provided evidence that transplanted hUCB-MNCs play therapeutic effects in a rat PD model by differentiating to neurons and dopamine neurons.
\end{abstract}

Keywords: hUCB-MNCs, Parkinson's disease, transplantation, differentiation, dopamine neurons

\section{Introduction}

Parkinson's disease (PD) is characterized by dopamine neuron loss in substantia nigra of patients' brains. Cell-based therapy represents a promising therapeutic approach to replace the missing dopamine neurons and restore the motor function of patients with PD. The major challenge for the development of stem cell replacement therapies for $\mathrm{PD}$ is to find reliable sources of easily expandable cells which can generate specific dopamine neurons that are lost in the brains of PD patients. ${ }^{1,2}$ In the past decades, different sources of stem cells have been investigated for the treatment of PD. ${ }^{3,4}$ Studies using fetal brain-derived neural stem cells (NSCs) and human embryonic stem cells (hESCs) have been shown to have therapeutic effects in animal PD models and in some patients with PD., ${ }^{3,5}$ Some studies showed that transplantation of human induced pluripotent stem cells (hiPSC)-derived NSCs and dopamine neurons promoted the functional recovery of motor deficits of the PD animal models. ${ }^{8,9}$ However, the hESC and fetal brain NSC have some ethical and immune-rejection issues, and the hiPSC may have the tumorigenic problems when these cells are used in clinical practice.

Recent studies have indicated that mononuclear cells of human umbilical cord blood (hUCB-MNCs) can improve the functional deficits of neurological diseases including 
stroke and traumatic brain injury in animal models. ${ }^{10-12}$ Mononuclear cells derived from hUCB-MNCs are easily and economically collected and are enriched in several classes of stem cells including endothelial progenitor cells and mesenchymal stem cells (MSCs), making hUCB-MNCs a promising cell for the treatment of degenerative disorders. ${ }^{13-15}$ The $\mathrm{MNC}$ fraction of hUCB was reported to contain hematopoietic stem cells (HSCs), endothelial stem cells, lymphoblasts, leukocytes, myeloid cells, and some small embryonic stem cells. ${ }^{16,17}$ Several studies have shown that hUCB-MNCs also contain NSCs and can promote neural regeneration and tissue repair in various neurological diseases, ${ }^{18-20}$ suggesting the therapeutic potential of hUCB-MNCs for the treatment of neurodegenerative diseases. Some studies showed that bone marrow-derived mesenchymal stem cells (BM-MSCs) had neuroprotective effects for PD in animal models and patients. ${ }^{21-23}$ In this study, we characterized the hUCB-MNCs and transplanted hUCB-MNCs to the rat PD model to study the therapeutic effects of hUCB-MNCs on PD.

\section{Materials and methods \\ Isolation and characterization of hUCB-MNCs and MSCs}

hUCB samples were obtained from healthy full-term newborns immediately after delivery, and this was done in accordance with the ethical guidelines of the Institutional Review Board at The Liaocheng People's Hospital, Affiliated Liaocheng Hospital, Taishan Medical University, Shandong, People's Republic of China; who also gave ethical approval for this study. Informed consent forms were completed and signed by donor mothers before hUCB samples were collected. The total MNC fraction was isolated from UC blood by Ficoll density gradient separation as described previously. ${ }^{24,25}$ The isolated hUCB-MNCs were then characterized by flow cytometry or used to isolate the MSC for neural differentiation or cryopreserved and stored in liquid nitrogen for long-term use.

To isolate the MSCs from hUCB-MNCs, we modified the previously reported procedures. ${ }^{26,27}$ Firstly, $2 \times 10^{7}$ of the mononuclear cells from hUCB-MNCs were plated onto T75 culture flasks and cultured in the media containing Dulbecco's Modified Eagle's Medium (DMEM)/F12, 10\% fetal bovine serum (FBS), $200 \mathrm{mmol} / \mathrm{L}-$ Glutamax, 1\% nonessential amino acid (NEAA), 1\% Pen-Strep (P/S), and basic fibroblast growth factor (bFGF; $5 \mu \mathrm{g} / \mathrm{mL}$ ). The nonadherent cells were removed after 48 hours of culture, and were replenished with fresh medium. Subsequently, the medium was replenished every 48 hours. After 10-14 days, MSCs were grown out in the culture flasks. Once the cells became confluent, they were dissociated with TrypLE and further expanded to provide the required number of cells for the experiments.

\section{Flow cytometry analysis}

The hUCB-MNCs were analyzed with a FACS Calibur flow cytometer (BD Biosciences, San Jose, CA, USA) immediately after isolation. A standard procedure was performed to characterize the hUCB-MNCs through the cell surface markers of CD16, CD29, CD34, CD45, CD73, and CD90 as we previously described. ${ }^{8}$ In brief, the hUCB-MNCs were suspended at a concentration of $1 \times 10^{7} / \mathrm{mL}$ in phosphatebuffered saline (PBS). Then $1 \times 10^{5}$ cells $(100 \mu \mathrm{L})$ were incubated with $50 \mu \mathrm{L}$ human serum for 15 minutes at room temperature (RT), washed with PBS, and centrifuged for 5 minutes at 1,000 rpm. Cell pellets were resuspended in $50 \mu \mathrm{L}$ PBS and incubated with $2 \mu \mathrm{L}$ fluorescein isothiocyanate (FITC)-conjugated anti-human CD90 or CD73 or CD16 or $2 \mu \mathrm{L}$ FITC-labeled IgG1 control isotype for 45 minutes at RT. For analysis of CD34, CD29, and CD45, cells were incubated with $2 \mu \mathrm{L}$ R-phycoerythrin (PE)-conjugated antihuman CD34 or CD29, or CD45 or $2 \mu \mathrm{L}$ PE-labeled IgG1 control isotype for 45 minutes at RT. After being washed with $2 \mathrm{~mL}$ PBS and centrifuged for 5 minutes at 1,000 rpm at RT, cells were suspended in $400 \mu \mathrm{L}$ PBS and loaded on a BD FACSAria II flow cytometer (BD Biosciences). We also analyzed the expressions of CD34, CD73, and CD90 for the MSCs of hUCB-MNCs.

\section{Neural differentiation of hUCB-MNCs- derived MSCs in vitro}

Culture flasks or dishes were precoated with poly-L-ornithine and mouse laminin (Sigma-Aldrich, St. Louis, MO, USA). After isolation, hUCB-MNCs were immediately plated and cultured in $\mathrm{T} 75$ flasks with differentiation medium containing DMEM/F12, supplemented with 20\% FBS, 1\% NEAA, 1\% glutamine, and $1 \% \mathrm{P} / \mathrm{S}$ with addition of $\mathrm{bFGF}(10 \mathrm{ng} / \mathrm{mL})$, epidermal growth factor (EGF) $(10 \mathrm{ng} / \mathrm{mL})$ for 2-4 weeks to isolate the MSCs. ${ }^{28}$ These MSCs were collected, subcultured, and passaged every 7-10 days after the cells grew and reached a confluency of $70 \%-80 \%$. These MSC cells were induced for neural differentiation by modifying the published procedure. ${ }^{23}$ In brief, our method was composed of three stages of differentiation. Stage one of inducing hUCB-MNCs-derived MSCs toward a neural fate: for the first 3 days, cells were plated in 6-well plates precoated with poly-ornithine and laminin (Sigma-Aldrich) at $3 \times 10^{5}$ cells/well and cultured in DMEM/ F12, N2, P/S, FGF-2 (10 ng/mL) and EGF (10 ng/mL). Stage two of inducing midbrain neuron specification: for the next 
10 days cells were switched to medium containing DMEM/ F12, B27 (2\%), N2 (1\%), P/S, Sonic Hedgehog (SHH, 100 ng/mL; R\&D Systems, Minneapolis, MN, USA), FGF-8 (10 ng/mL, PeproTech, Inc, Rocky Hill, NJ, USA), and AAP (200 mM, Sigma-Aldrich). Stage three of inducing maturation toward dopaminergic neurons: for the last 7-10 days medium was changed to DMEM/F12, B27(2\%), P/S, GDNF (50 ng/mL), BDNF (50 ng/mL; R\&D Systems), cAMP, and ascorbic acid (200 mM). All growth factors were purchased from R\&D Biosystems, Peprotech Inc, or Sigma-Aldrich. Cells were fixed for immunocytochemical characterization after 2-3 weeks of differentiation.

\section{Generation PD rat model and cell transplantation 6-OHDA-lesioned PD rats}

All animal experiments were carried out in accordance with local ethical guidelines and approval of the Animal Care and Use Committee in Liaocheng People's Hospital/The Affiliated Liaocheng Hospital, Taishan Medical University, People's Republic of China. Male Sprague-Dawley rats (180-200 g) were purchased from Weitonglihua biotechnology company (Beijing, People's Republic of China) and were deeply anesthetized with $1 \%$ isoflurane mixed in oxygen. 6-OHDA (6-hydroxidopamine, H4381; SigmaAldrich) at a concentration of $4 \mu \mathrm{g} / \mu \mathrm{L}$ was dissolved in PBS with $0.2 \mathrm{mg} / \mathrm{mL}$ ascorbic acid, then $4 \mu \mathrm{L}$ of 6 -OHDA was injected into the right substantia nigra of the rat midbrains at the rate of $1 \mu \mathrm{L} /$ minute at the anterior-posterior (AP) location, $-2.0 \mathrm{~mm}$, lateral $(\mathrm{L}),+2.5 \mathrm{~mm}$, vertical $(\mathrm{V}),-7.8 \mathrm{~mm}$ from the bregma of the rats with a KOPF instrument (David Kopf Instruments, Tujunga, CA, USA http://www.kopfinstruments.com/). The needle of the Hamilton syringe was kept in for 10 minutes after injection to prevent backfilling along the injection tract. After surgery, the animals were kept in the animal house to allow for recovery, and motor behavior testing was performed 2-4 weeks after 6-OHDA was injected into the rats.

\section{Cell transplantation}

After isolation from whole blood, the hUCB-MNCs were immediately diluted in PBS to yield a cell concentration of $2 \times 10^{5}$ cells $/ \mu \mathrm{L}$, then $5 \mu \mathrm{L}$ of cell suspension or PBS was transplanted into the striatum $(\mathrm{AP}=+0.7 \mathrm{~mm}, \mathrm{~L}=+3 \mathrm{~mm}, \mathrm{~V}=-5$ $\mathrm{mm}$ ) of the 6-OHDA-lesioned rats using a Hamilton syringe $(0.3-0.5 \mathrm{~mm}$ in diameter) over a period of 5-10 minutes. To avoid immune rejection of the transplanted cells, cyclosporine A (10 mg/kg; Sigma-Aldrich) was injected (intraperitoneal)
24 hours before transplantation and every day afterward until the rats were sacrificed for other analysis. To exclude the toxicological effects of cyclosporine A on PD rats, it was also given to the control group of the PD rats transplanted PBS at the same dosage as used in cell-transplanted PD rats.

\section{Motor behavior testing}

Apomorphine-induced rotations were measured to record the turning-over turns of the rats 3 minutes after apomorphine was injected into rats subcutaneously. The number of complete $\left(360^{\circ}\right)$ turns in a 30 -minute period was counted. Since the motor dysfunction appeared 2-4 weeks after 6-OHDA injection, rats with more than 200 asymmetric rotations in 30 minutes in response to apomorphine 4 weeks after 6-OHDA injection were used as recipients for cell transplantation and PBS control. In total, eight rats were transplanted with hUCB-MNCs (transplantation group) and seven rats were transplanted with PBS (control group). The ratio of the number of turns after transplantation versus the number of turns before transplantation was calculated and compared between the group of rats with transplanted cells and the control group with PBS injection at 4, 8, 12, and 16 weeks after transplantation.

\section{Immunocytochemistry and immunohistochemistry}

Fluorescence immunocytochemistry was performed as described previously. ${ }^{8,29}$ For differentiated cells, cells were fixed in 4\% paraformaldehyde in PBS for 20 minutes and were incubated in a blocking buffer (5\% goat serum, $5 \%$ donkey serum, and $0.2 \%$ triton $\mathrm{X}-100$ in PBS) for 45 minutes at RT. The fixed cells were then incubated with primary antibodies for 3-4 hours at RT, followed by incubation with fluorescence-labeled secondary antibody of Alexa Fluor 488-goat anti-mouse or Cy3-goat anti-rabbit (1:1,000, Jackson Laboratory, Sacramento, CA, USA), and then nuclear counterstaining with Hoechst 33258 for 2-3 minutes (1:10,000, Sigma-Aldrich). The primary antibodies were as follows: Nestin (1:500, mouse monoclonal, Sigma-Aldrich); Tuj1 (1:500, mouse monoclonal, Sigma-Aldrich), TH (1:250, rabbit polyclonal, Pel-Freeze Arkansas LLC, St, Rogers, AR, USA), GFAP (1:250, rabbit polyclonal, Dako, Carpinteria, CA, USA), and CD44 (1:500, mouse monoclonal, Sigma-Aldrich). Staining was visualized with a Nikon fluorescence microscope (Nikon Ti, Tokyo, Japan).

For immunohistochemistry, the rats were sacrificed at 16 weeks after cell transplantation and were perfused with 
4\% PFA (paraformaldehyde). The whole rat brains were removed and fixed in 4\% PFA for 24 hours. Subsequently, brains were cryoprotected in 30\% sucrose for 24-48 hours, embedded in Tissue-Tek OTC (Sakura Finetek Inc., Torrance, CA, USA), and sectioned into $20 \mu \mathrm{m}$ slices using a cryostat (Leika CM1950, Leica, Wetzlar, Germany). One in five sections were immunostained for human nucleus antibody (hNUC, mouse monoclonal, 1:500, Millipore, Billerica, MA, USA), human neuronal antibody (Tuj1, mouse monoclonal, 1:500, Sigma-Aldrich), dopamine neuron antibody (TH, Rabbit polyclonal, 1:250, Pel-Freez), and Glial antibody (GFAP, rabbit polyclonal 1:250, Dako). The primary antibody was incubated at $4^{\circ} \mathrm{C}$ overnight. After washing with PBS, the fluorescence-labeled secondary antibody of Alexa Fluor 488-goat anti-mouse or Cy3-goat anti-rabbit (1:1,000, Jackson Laboratories) was incubated for 3-4 hours and then incubated with Hoechst 33528 (1:10,000, Sigma-Aldrich) for 2-5 minutes. All images were taken using a fluorescence microscope (Nikon Ti).

\section{Statistical analysis}

All the data were statistically analyzed using the Student's $t$-test or one-way analysis of variance (ANOVA, nonparametric; SPSS statistics software 13.1, SPSS, Chicago, IL, USA).

\section{Results}

\section{Characterization of hUCB-MNCs by flow cytometry}

Since hUCB-MNCs are heterogeneous and contain different cells including lymphocytes, monocytes, HSCs, MSCs, and endothelial progenitor cells (EPCs), we analyzed the expression of surface antigens of hUCB-MNCs to know their cell compositions. After isolation of hUCB-MNCs, we immediately divided the cells into two parts. One part was frozen down and stored in liquid nitrogen. Another part was immediately used for flow cytometry analysis. We analyzed each of the three hUCB-MNC samples from three individual UC bloods. As a result, the average expression of CD16, CD29, CD34, CD45, CD73 and CD90 account for $31.90 \% \pm 3.67 \%, 71.50 \% \pm 0.56 \%, 3.05 \% \pm 1.06 \%$, $91.65 \% \pm 9.69 \%, 18.90 \% \pm 4.38 \%$, and $43.70 \% \pm 0.42 \%$ of the hUCB-MNC cells, respectively, indicating most of the cells are lymphocytes and monocytes expressing CD45, CD29, and CD90, but the hUCB-MNC showed very low expression of hematopoietic progenitor marker of CD34 as shown in Figure 1.

\section{Isolation and subculture of MSCs from hUCB-MNCs}

After isolation of hUCB-MNCs, $2 \times 10^{7}$ mononuclear cells were plated in T75 flasks for isolation of MSCs. During the culture, nonadherent cells were removed and fresh medium was added every 2 days. At 7 days, some MSCs were sparsely seen in the flasks as shown in Figure 2A-C. These primary MSCs were continuously cultured for 2-3 weeks to reach confluency. After that, cells were passaged every week to get the P1 and P2 subcultures for neural differentiation or were stored in liquid nitrogen. To characterize the MSCs, we performed flow cytometry analysis and found that these MSCs expressed the surface markers of CD73 (87.6\% $\pm 7.48 \%)$, CD90 (94.5\% $\pm 5.81 \%)$, and were almost absent of CD34 $(0.4 \% \pm 0.12 \%)$, which was similar to that in UC-derived MSCs. ${ }^{30}$ We also used the immunostainning to examine the expression of CD44 and found that the MSCs highly expressed CD44 (93.6\% $\pm 5.23 \%)$ as shown in Figure 2D-F.

\section{Neural differentiation potential of hUCB-MNCs}

On the basis of the previously published protocols to differentiate MSCs and iPS cells to neurons and dopamine neurons, ${ }^{8,23,31}$ we used three-stage protocol to induce the hUCB-MNCs-derived MSCs to neurons and dopamine neurons (Figure 3 ).

After 3 weeks of induction, the cells were characterized by morphological changes and by immunostaining. We found that the cells change to neuronlike cells after 2 weeks and stained with neuronal antibodies of Tuj1 and MAP2, as well as the glial antibodies of GFAP. This indicated that the hUCB-MNC-derived MSCs were able to be induced to neurons and astrocytes, but no cells were stained for TH and DAT, suggesting that dopamine neurons were not able to be induced from hUCB-MNCs in our in vitro experiments. Some of the cells were stained with Nestin, indicating some cells differentiated to NSCs (Figure 4).

\section{Neurorestorative effects of transplanted hUCB-MNCs in 6-OHDA-induced $P D$ rats}

We injected the 6-OHDA to substantia nigra to induce the rat PD model. The 6-OHDA-injected rats were screened for motor impairment by measuring the turning-over behavior. The rats were selected for cell transplantation if they had more than 200 apomorphine-induced turning-overs in 30 minutes. Totally, 16 PD rats were enrolled for transplantation study. Eight rats received hUCB-MNCs to the striatum of the rats (hUCB-MNCs group), while the other 8 rats were injected 
A

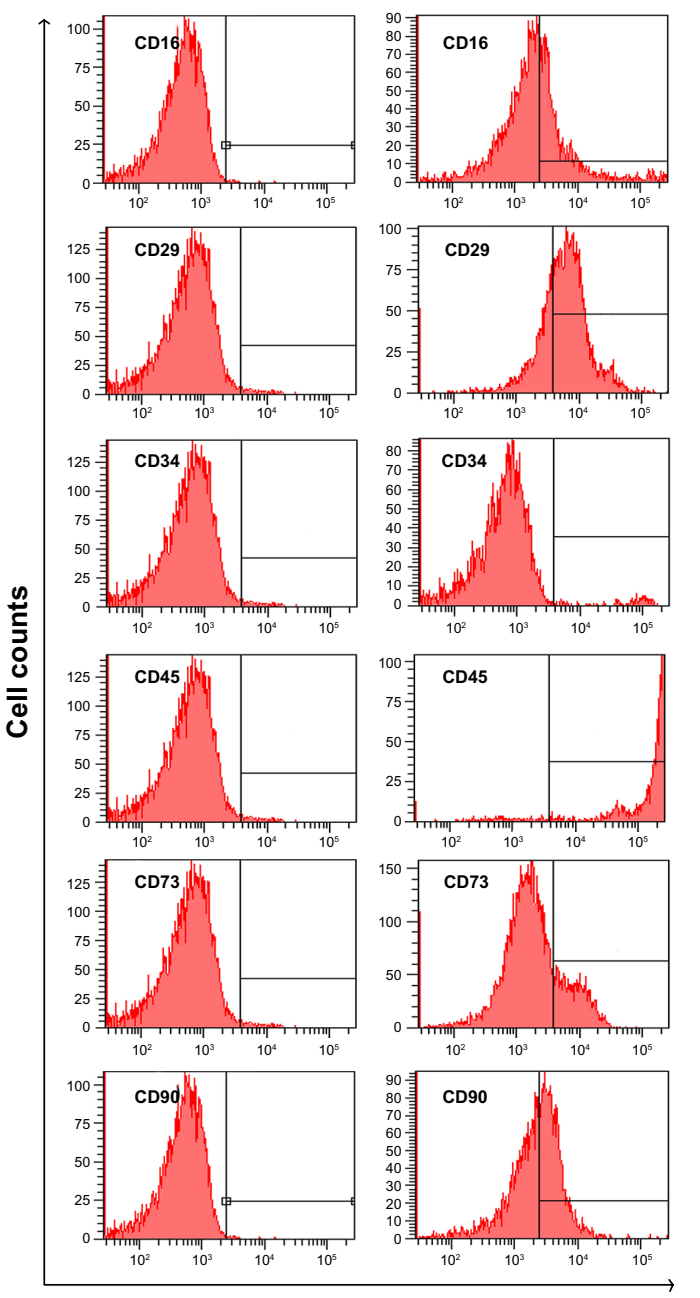

B

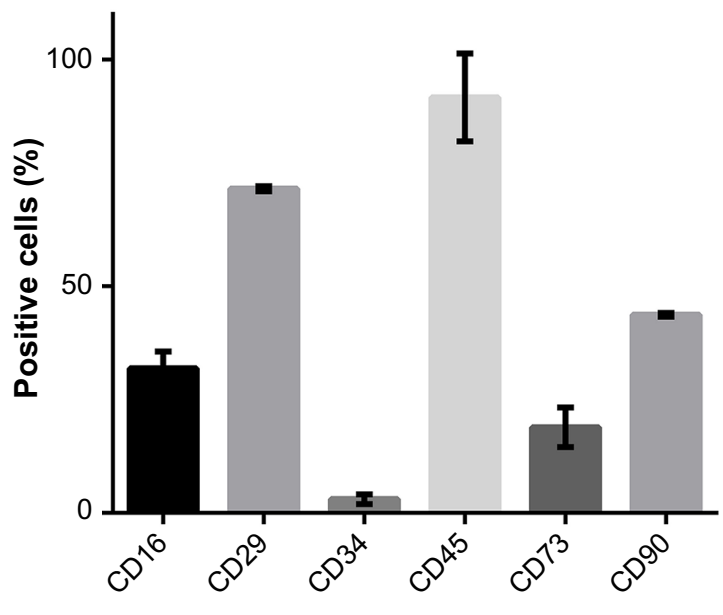

Fluorescence

Figure I Flow cytometry analysis of the hUCB-MNCs.

Notes: (A) Expression of surface markers of hUCB-MNCs from one individual. (B) The average expression rates of CDI6, CD29, CD34, CD45, CD73, and CD90 in hUCB-MNCs from three individuals.

Abbreviation: hUCB-MNCs, human umbilical cord blood-derived mononuclear cells.
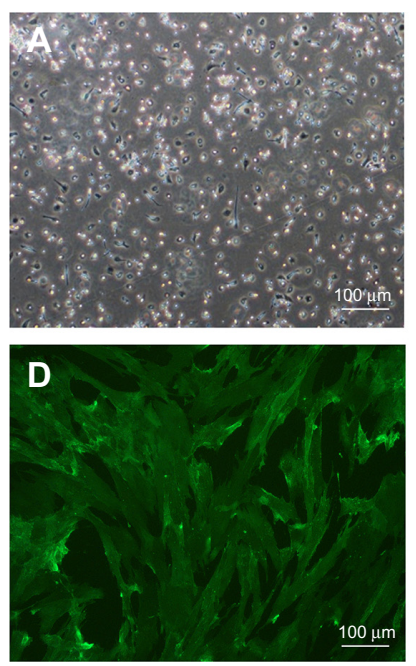
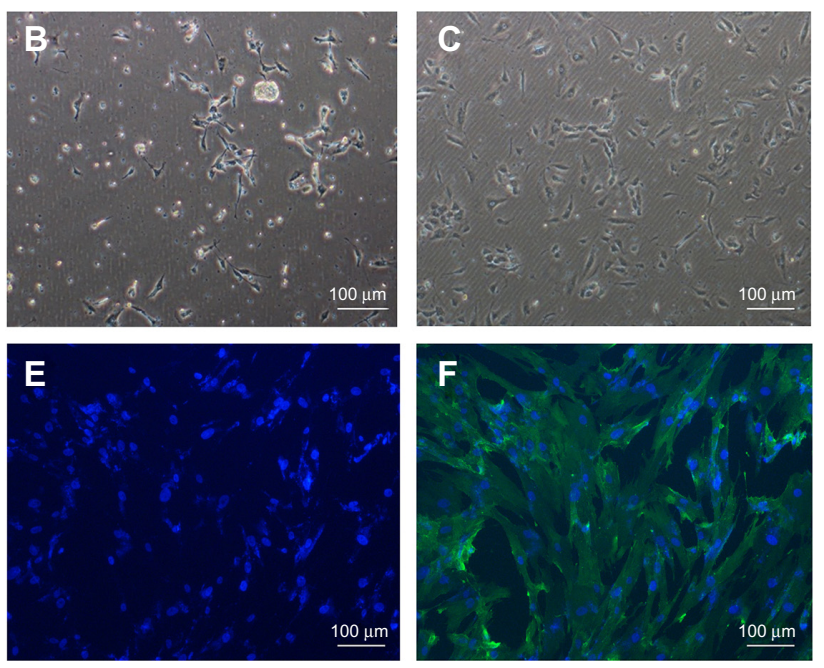

Figure 2 Isolation and characterization of MSCs from hUCB-MNCs.

Notes: Morphologies of MSCs derived from hUCB-MNCs after 7, 14, and 21 days of culturing (A-C), respectively. Immunostaining of cultured MSCs with CD44 (D), Hoechst 33258 (E), and merged CD44/Hoechst 33258 (F).

Abbreviations: MSCs, mesenchymal stem cells; hUCB-MNCs, human umbilical cord blood-derived mononuclear cells. 


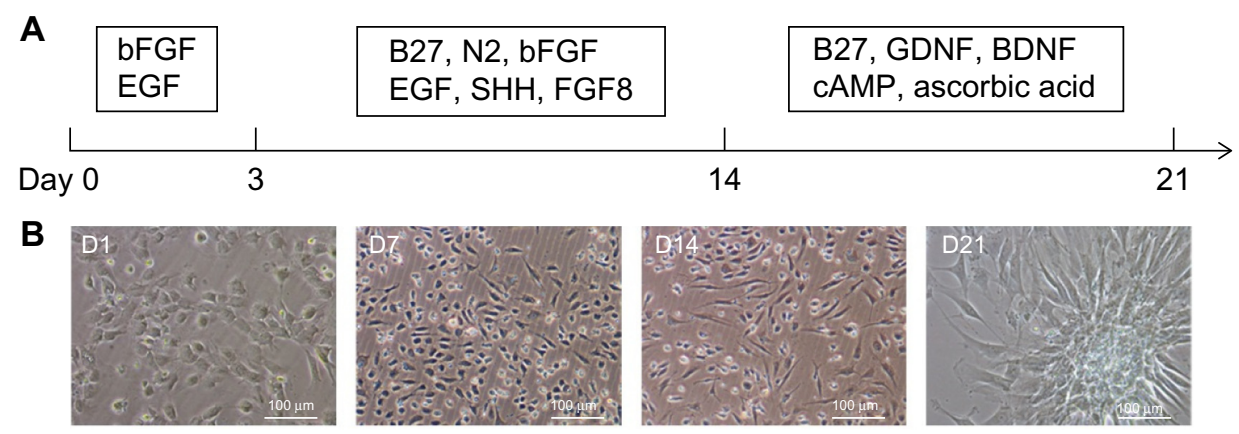

Figure 3 In vitro neural differentiation of hUCB-MNC-derived MSCs.

Notes: (A) Our modified protocol to differentiate MSCs of hUCB-MNCs to neurons. (B) Bright-field images of differentiated MSCs of hUCB-MNCs at different stages (magnification $\times 40$, scale bar $=100 \mu \mathrm{m}$ ).

Abbreviations: bFGF, basic fibroblast growth factor; D, day; EGF, epidermal growth factor; hUCB-MNCs, human umbilical cord blood-derived mononuclear cells; MSCs, mesenchymal stem cells.
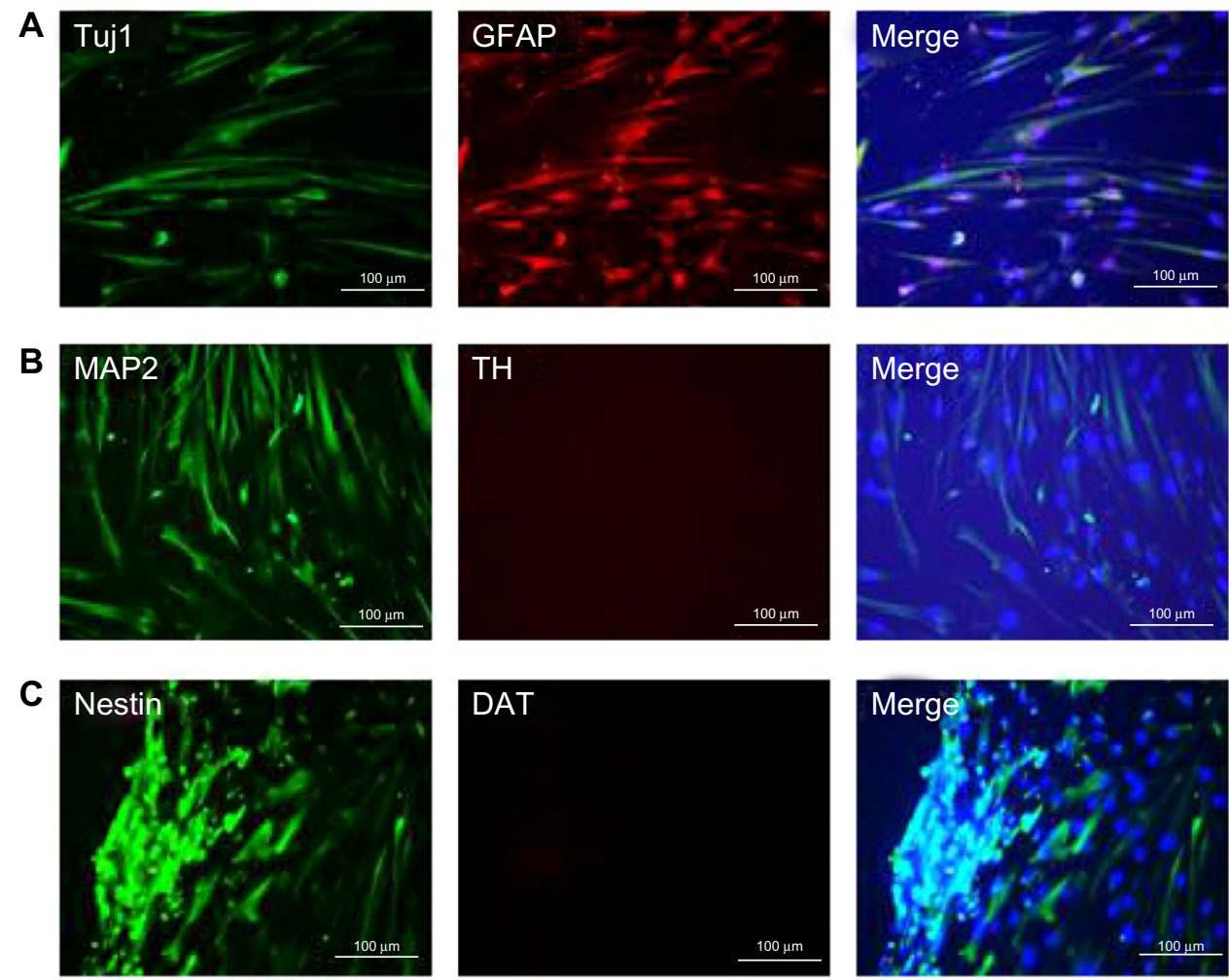

D

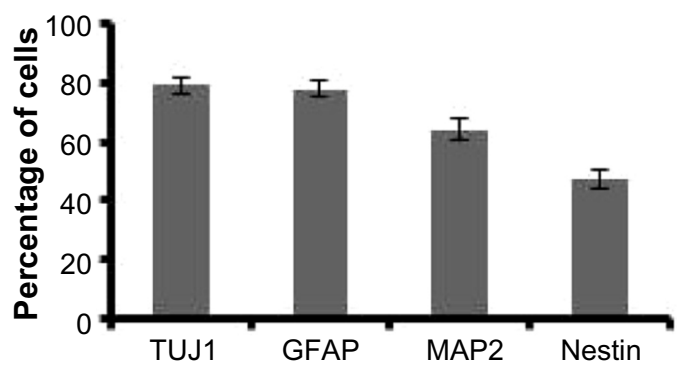

Figure 4 Immunostaining analysis for in vitro differentiated cells of hUCB-MNCs.

Notes: (A) Differentiated neurons and astrocytes stained with neuronal marker Tuj I and astrocyte marker GFAP (magnification $\times 100$, scale bar $=100 \mu \mathrm{m})$. (B) Differentiated neurons stained with neuronal marker MAP2 and dopamine neuron marker TH (magnification $\times 100$, scale bar $=100 \mu \mathrm{m}$ ). (C) Differentiated cells of hUCB-MNC-derived MSCs stained with NSC marker Nestin and dopamine neuron marker DAT (magnification $\times 100$, scale bar $=100 \mu \mathrm{m}$ ). (D) Quantification of differentiated neurons (Tuj I/MAP2 staining), astrocytes (GFAP staining), and NSCs from hUCB-MNCs-derived MSCs (Nestin staining).

Abbreviations: hUCB-MNCs, human umbilical cord blood-derived mononuclear cells; MSCs, mesenchymal stem cells; NSC, neural stem cell. 
with PBS without any cells (PBS group). One rat of the hUCB-MNCs group and two rats of the PBS group were taken out of the study because of their decreased health conditions. Thus, 7 rats of hUCB-MNCs group and 6 rats in PBS group were followed to the end of this study (16 weeks). We found that 6-OHDA-lesioned rats that received the hUCB-MNCs transplantation had significant reduction in rotational response to apomorphine compared to the $\mathrm{PD}$ rats transplanted with PBS (Figure 5). Immunosuppressive agent cyclosporine $\mathrm{A}$ was used at the dose of $10 \mathrm{mg} / \mathrm{kg}$ daily for all PD rats 2 days before transplantation until the end of the study.

\section{The survival and neural differentiation of grafted hUCB-MNCs in vivo}

To understand the survival, differentiation, and migration ability of transplanted hUCB-MNCs, the rats were perfused and their brains removed for immunohistochemistry after 16 weeks of transplantation. We used human-specific antibody of hNUC to label the transplanted human cells. The presence of the human-specific hNUC was observed in the transplanted 6-OHDa-lesioned striatum, suggesting the survival of human-derived cells. Immunohistochemistry analysis showed morphological changes of the transplanted hUCB-MNCs toward the neural lineages in host brains of the rats. Image analysis showed that hUCB-MNCs differentiated to neurons (Tuj1 staining), dopamine neurons (TH staining), and astrocytes (GFAP staining; Figure 6). Some of grafted

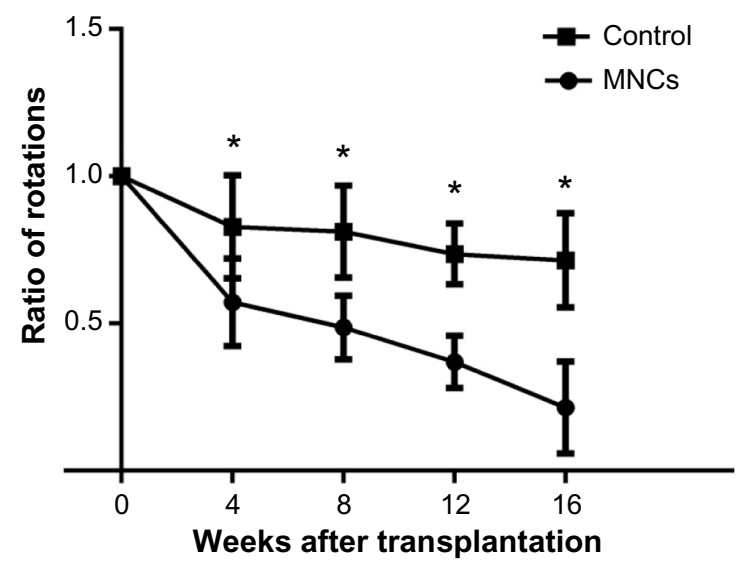

Figure 5 Comparison of motor behavior assessments between the 6-OHDAinduced rats with grafted MNCs and sham (control).

Notes: The frequency of amphetamine-induced rotations (turning-over) is significantly reduced in rats with transplanted MNCs (MNCs line) compared with control rats (PBS injection) at 12 and 16 weeks after MNCs transplantation. Shown are the average ratios of turning-over in $\mathbf{3 0}$ minutes after amphetamine injection as well as their standard deviations. (*indicates $P<0.01$.)

Abbreviations: MNCs, mononuclear cells; PBS, phosphate-buffered saline.
hUCB-MNCs were found to be costained with hNUC and TH (Figure 6B) or hNUC and GFAP (Figure 6C), indicating that these dopamine neurons and astrocytes are derived from transplanted hUCB-MNCs. To examine the microglial differentiation ability of the transplanted hUCB-MNCs in vivo, we stained the brain sections with microglial antibody of CD11b, but no cells were stained with CD11b, suggesting that no microglial cells were differentiated from the transplanted hUCB-MNCs in this study (Figure 6D). We also used the Western blot to analyze if immune responsive factors of TNF- $\alpha$ and TNF- $\beta$ are released from transplanted hUCB-MNCs, but neither TNF- $\alpha$ nor TNF- $\beta$ were detected in the brain homogenates of rats with transplanted cells (data not shown).

\section{Discussion}

A recent study showed that CD45-positive peripheral blood insulin-producing cells could be induced into astrocyte-like cells, and this unique CD45-positive cell pool may have a protective role in some degenerative diseases of the central nervous system. ${ }^{32}$ But no study has shown that hUCBMNCs have therapeutic effects on PD in animal models. Here we found there is a significant improvement of the motor dysfunction behaviors in 6-OHDA-induced PD rats through transplantation of hUCB-MNCs into the striatum of rat brains. We observed the recovery of motor deficits in 6-OHDA-lesioned rats from 4 through 16 weeks after transplantation of the hUCB-MNCs into the rats. This effect could be attributed to the differentiated neurons of grafted hUCBMNCs as we observed human cell-derived neurons and dopamine neurons in the brain sections of the transplanted rats, which was evidenced by the human positive-TH cells in the transplanted rat brains.

Some studies suggested that the transplanted stem cells may secrete neurotrophic growth factors such as BDNF, GDNF, or IGF or immune response factors such as the IL-2, $6,8,10,22,33$ we measured BDNF and GDNF using ELISA kits from Promega Corporation but did not detect any of these two factors in the sections of the rat brains (data not shown). We assumed that the motor improvement of PD rats might be partially increased by the immunomodulation and production of the growth factors secreted by the transplanted cells, even though we did not detect these factors in the transplanted rats. But we do not think the growth factors secreted by transplanted $\mathrm{MNC}$ cells play major roles in recovering motor deficits of the 6-OHDA-lesioned rats as the improvement of the motor behaviors was not seen until 4 weeks 

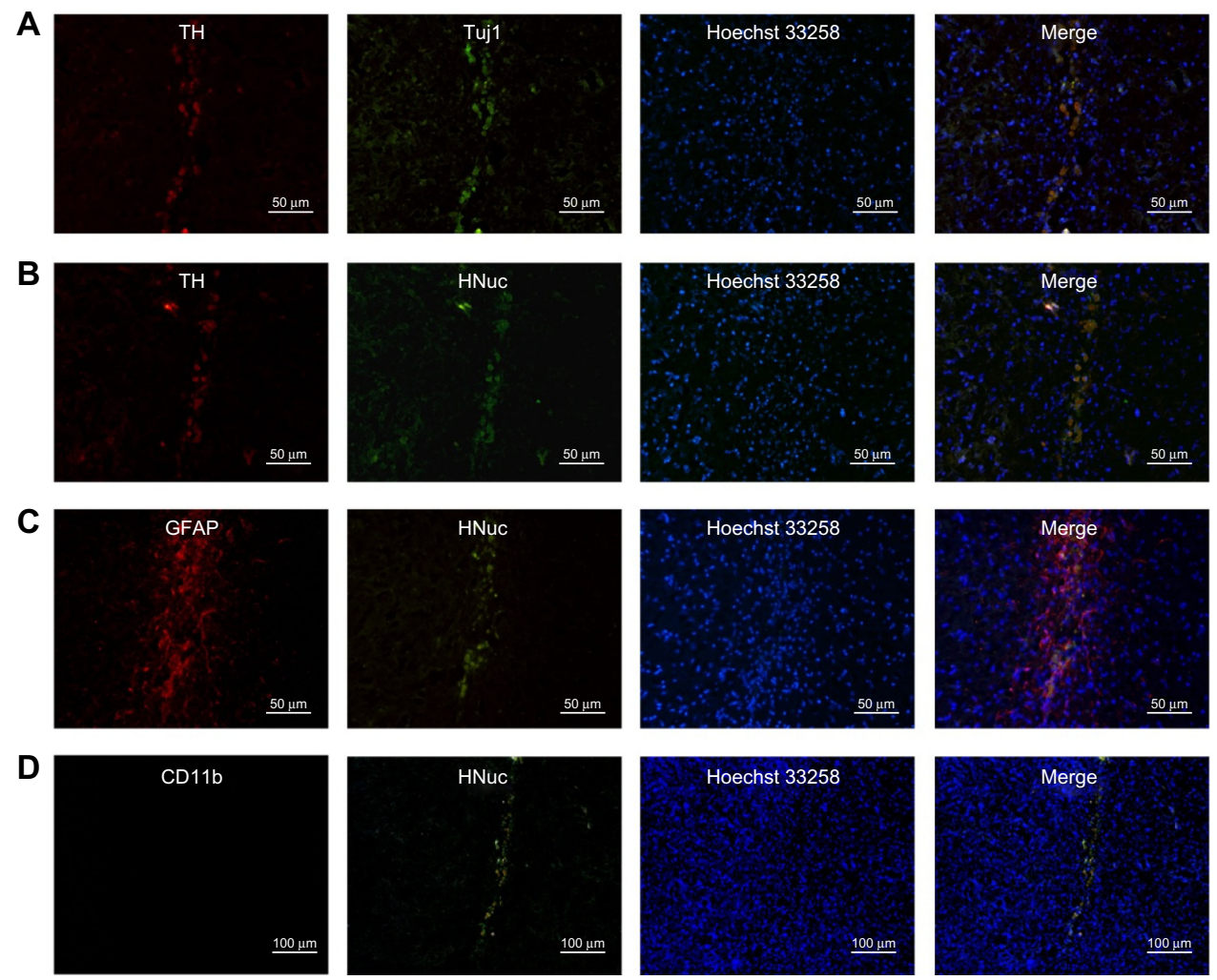

Figure 6 Immunohistochemistry analysis on the middle brains of 6-OHDA-induced rats with grafted MNCs.

Notes: (A) hUCB-MNCs grafts immunostained for TH (red), Tujl (green), or Hoechst 33258 (blue) at I6 weeks after transplantation. (B) hUCB-MNCs grafts immunostained for TH (red), human nuclear antigen (green), and Hoechst 33258 (blue) at 16 weeks after transplantation. (C) hUCB-MNCs grafts immunostained for GFAP (red), HNuc (green), and Hoechst 33258 (blue) at 16 weeks after transplantation. (D) hUCB-MNCs grafts immunostained for CDI Ib (red), HNuc (green), and Hoechst 33258 (blue) at 16 weeks after transplantation. (A-C: magnification $\times 100$, scale bar $=50 \mu \mathrm{m}$; $\mathbf{D}$ magnification $\times 100$, scale bar $=100 \mu \mathrm{m}$.)

Abbreviation: hUCB-MNCs, human umbilical cord blood-derived mononuclear cells.

after transplantation (data not shown). Nevertheless, some studies reported that the transplanted NSCs overexpressing GDNF or BDNF or NGF improved the locomotive or memory deficits of the animal models with PD, amyotrophic lateral sclerosis, and Alzheimer's disease, probably because of secreted growth factors playing major neurorestorative effects in these studies. ${ }^{29,34-36}$

The compositions of the hUCB-MNCs are still not uniform. One study showed that the hUCB-MNCs contain different stem cells and CD34/CD45 is about $60 \% .{ }^{24}$ In our study, most of the hUCB-MNCs are CD45-/CD29-positive, indicating that most of the cells in hUCB-MNCs are lymphocytes and monocytes. We found that CD34 is only $4.8 \%$ of hUCB-MNCs. This difference needs to be further validated by analyzing more samples of hUCB-MNCs.

Human MSCs can be isolated from different organs or tissue compartments such as the bone marrow, umbilical cord, UCB, placenta, adipose tissue, and many others. . $^{37,38}$ Unlike other stem cell populations such as hESCs, most human MSCs in culture display a limited expansion potential in vitro and can only propagate for about $6-10$ passages.
For the characterization of MSCs of hUCB-MNCs, we found that these MSCs highly expressed CD44, CD73, and CD90 with less expression of CD34, which is consistent to that in other studies. ${ }^{39,40}$ These characteristics support that hUCB-MNC-derived MSCs may have the multipotential to differentiate to the mesoderm and ectoderm lineages including the neural differentiation.

Even though HSCs were also reported to differentiate to NSCs in the culturing medium supplemented with thrombopoietin, flt-3 ligand, and c-kit ligand (TPOFLK), ${ }^{41}$ no studies have shown that hUCB-MNCs were induced to neurons in vitro. As the MSCs were reported to differentiate to neurons, we characterized MSCs from hUCB-MNCs and were able to differentiate the hUCB-MNC-derived MSCs to the NSCs and neurons in vitro. This is probably because the addition of signal molecules of SHH and FGF8 and the neurotrophic growth factors of BDNF and GDNF increased the neuronal conversion of the hUCB-MNCs. However, no dopamine neurons were found from differentiated neurons of hUCB-MNCs in vitro, suggesting generation of dopamine neurons may need more specific induction conditions. How- 
ever, we have noticed that in hUCB-MNC-transplanted rat brains, some differentiated neural cells were stained with hNUC and TH antibodies, indicating these are dopamine neurons from transplanted hUCB-MNCs. Since no dopamine neuron differentiation of hUCB-MNCs was found in vitro, probably the host microenvironment of the rat brain promoted the production of dopamine neurons from hUCB-MNCs in vivo. Bachstetter et $\mathrm{al}^{42}$ found that a single intravenous injection of hUCB-MNCs in aged rats can significantly improve the microenvironment of the aged hippocampus and rejuvenate the aged neural stem/progenitor cells. They raise the possibility of a peripherally administered cell therapy as an effective approach to improve the microenvironment of the 6-OHDA-lesioned brain.

After transplantation of hUCB-MNCs into the striatum of the 6-OHDA-induced PD rats, these hUCB-MNCs can survive and differentiate into neurons and dopamine neurons to improve the asymmetry systems of 6-OHDA-induced PD rats. Our study provided solid experimental evidence that hUCB-MNCs replacement has therapeutic potential for PD. The therapeutic effect of our hUCB-MNCs is obvious because seven transplanted rats showed some improvement of the asymmetry behavior of 6-OHDA-induced rats at different times after transplantation. In addition, none of the seven transplanted rats died earlier than the time point of death, suggesting that local injection of hUCB-MNCs to the rat brain reached the appropriate location without inducing traumatic side effects.

In the previous study, we have noticed that the functional recovery of the behavior defect in PD rats occurred as early as 4 weeks and through 16 weeks after transplantation of iPS cell-derived NSCs. Moreover, we noticed that 6-OHDAinduced motor defects could be seen in approximately $70 \%$ of the injected rats and that most of the 6-OHDA-induced rats have observable motor defects 3-4 weeks after 6-OHDA injection. ${ }^{8}$ In this study, we found a similar functional recovery of the 6-OHDA-induced rat defects through administration of hUCB-MNCs.

In addition to neuronal differentiation of hUCB-MNCs, there are other possible mechanisms for the motor function improvement induced by hUCB-MNCs. Cells were able to prevent necrosis, ${ }^{43,44}$ increase immunomodulation, ${ }^{45}$ decrease peripheral inflammatory response ${ }^{46}$ or microglial response, ${ }^{47}$ and stimulate neurogenesis. ${ }^{42}$ Moreover, hUCB-MNCs subfractions were reported to be able to promote angiogenesis ${ }^{48}$ and may prevent oxidative damage. ${ }^{49}$ These processes can lead to an overall neuronal differentiation and behavioral improvements of the 6-OHDA-induced PD rats. However, the microglia differentiation and immune inflammatory factors may not be attributed to the functional improvements of the 6-OHDA-induced PD rats in our study because we did not find any microglial cells differentiated from transplanted hUCB-MNCs. In addition, we did not detect the secretion of TNF- $\alpha$ and TNF- $\beta$ from the transplanted hUCB-MNCs. This can be explained by two possibilities. One possibility is that the transplanted hUCB-MNCs did not secrete TNF- $\alpha$ and TNF- $\beta$ in our study. Another possibility is that the amount of the TNF- $\alpha$ and TNF- $\beta$ produced by transplanted hUCBMNCs is too low to be detected by Western blot or other immune responsive factors such as interleukins, which were not examined in this study. We will examine more immune inflammatory factors in future studies.

The use of hUCB-MNCs is a valuable source for cell therapy since it confers neurorestoration in PD animal models. In future studies, we would perform detailed analyses for the responsible subpopulations of hUCB-MNCs and the mechanisms involved. Moreover, the brain areas into which implanted cells have integrated into, the differentiated neuronal phenotypes, their axonal projections, the synaptic connections between the host and the grafted cells, and the electrophysiological functions of the transplanted hUCBMNCs would also be performed.

In conclusion, this study demonstrated that transplanted hUCB-MNCs have therapeutic effects in a rat PD model by differentiating to neurons and dopamine neurons. Our results suggest that the modulation of endogenous adult neurogenesis to repair the damaged PD brain using hUCBMNCs would have a significant impact on future strategies for PD treatment.

\section{Acknowledgments}

The authors thank all the participants in the study. Funding was provided through the Science and Technology Department of Shandong Province, Shandong, People's Republic of China (2012GSF11808).

\section{Disclosure}

The authors report no conflicts of interest in this work.

\section{References}

1. Zhu S, Zhao C, Wu Y, et al. Identification of a Vav2-dependent mechanism for GDNF/Ret control of mesolimbic DAT trafficking. Nat Neurosci. 2015;18(8):1084-1093.

2. Dawson TM, Ko HS, Dawson VL. Genetic animal models of Parkinson's disease. Neuron. 2010;66(5):646-661.

3. Barker RA, Barrett J, Mason SL, Bjorklund A. Fetal dopaminergic transplantation trials and the future of neural grafting in Parkinson's disease. Lancet Neurol. 2013;12(1):84-91.

4. Han F, Baremberg D, Gao J, et al. Development of stem cell-based therapy for Parkinson's disease. Transl Neurodegener. 2015;4:16. 
5. Lindvall O, Kokaia Z. Prospects of stem cell therapy for replacing dopamine neurons in Parkinson's disease. Trends Pharmacol Sci. 2009;30(5):260-267.

6. Yang D, Zhang ZJ, Oldenburg M, Ayala M, Zhang SC. Human embryonic stem cell-derived dopaminergic neurons reverse functional deficit in parkinsonian rats. Stem Cells. 2008;26(1):55-63.

7. Singh A, Gutekunst CA, Uthayathas S, et al. Effects of fibroblast transplantation into the internal pallidum on levodopa-induced dyskinesias in parkinsonian non-human primates. Neurosci Bull. 2015;31(6):705-713.

8. Han F, Wang W, Chen B, et al. Human induced pluripotent stem cellderived neurons improve motor asymmetry in a 6-hydroxydopamineinduced rat model of Parkinson's disease. Cytotherapy. 2015;17(5): 665-679.

9. Hargus G, Cooper O, Deleidi M, et al. Differentiated Parkinson patientderived induced pluripotent stem cells grow in the adult rodent brain and reduce motor asymmetry in Parkinsonian rats. Proc Natl Acad Sci USA. 2010;107(36):15921-15926.

10. Karlupia N, Manley NC, Prasad K, Schafer R, Steinberg GK. Intraarterial transplantation of human umbilical cord blood mononuclear cells is more efficacious and safer compared with umbilical cord mesenchymal stromal cells in a rodent stroke model. Stem Cell Res Ther. 2014;5(2):45.

11. Chen J, Sanberg PR, Li Y, et al. Intravenous administration of human umbilical cord blood reduces behavioral deficits after stroke in rats. Stroke. 2001;32(11):2682-2688.

12. Rodrigues LP, Iglesias D, Nicola FC, et al. Transplantation of mononuclear cells from human umbilical cord blood promotes functional recovery after traumatic spinal cord injury in Wistar rats. Braz J Med Biol Res. 2012;45(1):49-57.

13. Hows JM, Marsh JC, Bradley BA, et al. Human cord blood: a source of transplantable stem cells? Bone Marrow Transplant. 1992;9(Suppl 1): 105-108.

14. Yang WZ, Zhang Y, Wu F, et al. Safety evaluation of allogeneic umbilical cord blood mononuclear cell therapy for degenerative conditions. J Transl Med. 2010;8:75.

15. Sanberg PR, Eve DJ, Willing AE, et al. The treatment of neurodegenerative disorders using umbilical cord blood and menstrual blood-derived stem cells. Cell Transplant. 2011;20(1):85-94.

16. Weiss ML, Troyer DL. Stem cells in the umbilical cord. Stem Cell Rev. 2006;2(2):155-162.

17. Roussev RG, Higgins NG, McIntyre JA. Phenotypic characterization of normal human placental mononuclear cells. J Reprod Immunol. 1993;25(1):15-29

18. Domanska-Janik K, Buzanska L, Lukomska B. A novel, neural potential of non-hematopoietic human umbilical cord blood stem cells. Int J Dev Biol. 2008;52(2-3):237-248.

19. Zangiacomi V, Balon N, Maddens S, et al. Human cord blood-derived hematopoietic and neural-like stem/progenitor cells are attracted by the neurotransmitter GABA. Stem Cells Dev. 2009;18(9):1369-1378.

20. Rosenkranz K, Meier C. Umbilical cord blood cell transplantation after brain ischemia - from recovery of function to cellular mechanisms. Ann Anat. 2011;193(4):371-379.

21. Venkataramana NK, Kumar SK, Balaraju S, et al. Open-labeled study of unilateral autologous bone-marrow-derived mesenchymal stem cell transplantation in Parkinson's disease. Transl Res. 2010;155(2): 62-70.

22. Blandini F, Cova L, Armentero MT, et al. Transplantation of undifferentiated human mesenchymal stem cells protects against 6-hydroxydopamine neurotoxicity in the rat. Cell Transplant. 2010;19(2): 203-217.

23. Khoo ML, Tao H, Meedeniya AC, Mackay-Sim A, Ma DD. Transplantation of neuronal-primed human bone marrow mesenchymal stem cells in hemiparkinsonian rodents. PLoS One. 2011;6(5):e19025.

24. Weise G, Lorenz M, Posel C, et al. Transplantation of cryopreserved human umbilical cord blood mononuclear cells does not induce sustained recovery after experimental stroke in spontaneously hypertensive rats. J Cereb Blood Flow Metab. 2014;34(1):e1-e9.
25. Jaatinen T, Laine J. Isolation of mononuclear cells from human cord blood by Ficoll-Paque density gradient. Curr Protoc Stem Cell Biol. 2007; Chapter 2:Unit 2A 1.

26. Barlow S, Brooke G, Chatterjee K, et al. Comparison of human placenta- and bone marrow-derived multipotent mesenchymal stem cells. Stem Cells Dev. 2008;17(6):1095-1107.

27. Li X, Du W, Ma FX, et al. High concentrations of TNF-alpha induce cell death during interactions between human umbilical cord mesenchymal stem cells and peripheral blood mononuclear cells. PLoS One. 2015;10(5):e0128647.

28. Laitinen A, Nystedt J, Laitinen S. The isolation and culture of human cord blood-derived mesenchymal stem cells under low oxygen conditions. Methods Mol Biol. 2011;698:63-73.

29. Suzuki M, McHugh J, Tork C, et al. GDNF secreting human neural progenitor cells protect dying motor neurons, but not their projection to muscle, in a rat model of familial ALS. PLoS One. 2007;2(8):e689.

30. Li S, Wang Y, Guan L, Ji M. Characteristics of human umbilical cord mesenchymal stem cells during ex vivo expansion. Mol Med Rep. 2015;12(3):4320-4325.

31. Chambers SM, Fasano CA, Papapetrou EP, et al. Highly efficient neural conversion of human ES and iPS cells by dual inhibition of SMAD signaling. Nat Biotechnol. 2009;27(3):275-280.

32. Li H, Li J, Sheng W, et al. Astrocyte-like cells differentiated from a novel population of CD45-positive cells in adult human peripheral blood. Cell Biol Int. 2015;39(1):84-93.

33. Danielyan L, Schafer R, von Ameln-Mayerhofer A, et al. Therapeutic efficacy of intranasally delivered mesenchymal stem cells in a rat model of Parkinson disease. Rejuvenation Res. 2011;14(1):3-16.

34. Fan X, Sun D, Tang X, et al. Stem-cell challenges in the treatment of Alzheimer's disease: a long way from bench to bedside. Med Res Rev. 2014;34(5):957-978.

35. Lee HJ, Lim IJ, Park SW, et al. Human neural stem cells genetically modified to express human nerve growth factor (NGF) gene restore cognition in the mouse with ibotenic acid-induced cognitive dysfunction. Cell Transplant. 2012;21(11):2487-2496.

36. Liu TW, Ma ZG, Zhou Y, Xie JX. Transplantation of mouse CGR8 embryonic stem cells producing GDNF and TH protects against 6-hydroxydopamine neurotoxicity in the rat. Int J Biochem Cell Biol. 2013;45(7):1265-1273.

37. Meirelles Lda S, Nardi NB. Methodology, biology and clinical applications of mesenchymal stem cells. Front Biosci (Landmark Ed). 2009;14:4281-4298.

38. Bianco P, Robey PG, Simmons PJ. Mesenchymal stem cells: revisiting history, concepts, and assays. Cell Stem Cell. 2008;2(4):313-319.

39. Otsuru S, Hofmann TJ, Olson TS, Dominici M, Horwitz EM. Improved isolation and expansion of bone marrow mesenchymal stromal cells using a novel marrow filter device. Cytotherapy. 2013;15(2):146-153.

40. Dominici M, Le Blanc K, Mueller I, et al. Minimal criteria for defining multipotent mesenchymal stromal cells. The International Society for Cellular Therapy position statement. Cytotherapy. 2006;8(4):315-317.

41. McGuckin CP, Forraz N, Allouard Q, Pettengell R. Umbilical cord blood stem cells can expand hematopoietic and neuroglial progenitors in vitro. Exp Cell Res. 2004;295(2):350-359.

42. Bachstetter AD, Pabon MM, Cole MJ, et al. Peripheral injection of human umbilical cord blood stimulates neurogenesis in the aged rat brain. BMC Neurosci. 2008;9:22.

43. Boltze J, Schmidt UR, Reich DM, et al. Determination of the therapeutic time window for human umbilical cord blood mononuclear cell transplantation following experimental stroke in rats. Cell Transplant. 2012;21(6):1199-1211.

44. Button RW, Luo S, Rubinsztein DC. Autophagic activity in neuronal cell death. Neurosci Bull. 2015;31(4):382-394.

45. Sheikh AM, Nagai A, Wakabayashi K, et al. Mesenchymal stem cell transplantation modulates neuroinflammation in focal cerebral ischemia: contribution of fractalkine and IL-5. Neurobiol Dis. 2011;41(3):717-724. 
46. Leonardo CC, Hall AA, Collier LA, et al. Human umbilical cord blood cell therapy blocks the morphological change and recruitment of CD11b-expressing, isolectin-binding proinflammatory cells after middle cerebral artery occlusion. J Neurosci Res. 2010;88(6):1213-1222.

47. Jiang L, Womble T, Saporta S, et al. Human umbilical cord blood cells decrease microglial survival in vitro. Stem Cells Dev. 2010;19(2): 221-228.
48. Vanneaux V, El-Ayoubi F, Delmau C, et al. In vitro and in vivo analysis of endothelial progenitor cells from cryopreserved umbilical cord blood: are we ready for clinical application? Cell Transplant. 2010;19(9):1143-1155.

49. Arien-Zakay H, Lecht S, Bercu MM, et al. Neuroprotection by cord blood neural progenitors involves antioxidants, neurotrophic and angiogenic factors. Exp Neurol. 2009;216(1):83-94.

\section{Publish your work in this journal}

The Journal of Neurorestoratology is an international, peer-reviewed, open access online journal publishing original research and review articles on the subject of Neurorestoratology. To provide complete coverage of this revolutionary field the Journal of Neurorestoratology will report on relevant experimental research, technological advances, and

\section{Dovepress}

clinical achievements. The manuscript management system is completely online and includes a very quick and fair peer-review system, which is all easy to use. Visit http://www.dovepress.com/testimonials.php to read real quotes from published authors.

Submit your manuscript here: http://www.dovepress.com/journal-of-neurorestoratology-journal 